\title{
Experimental study on mixed traffic flow of bicycles and pedestrians
}

\author{
Ning Guo', Rui Jiang ${ }^{2}$, SC Wong ${ }^{3}$, Qing-Yi Hao ${ }^{4}$, Shu-Qi Xue ${ }^{2}$, Yao Xiao ${ }^{2}$, Chao-Yun Wu \\ ${ }^{1}$ School of Automotive and Transportation Engineering, Hefei University of Technology \\ 230009, Hefei, P.R. China \\ guoning_945@126.com \\ ${ }^{2}$ Key Laboratory of Transport Industry of Big Data Application Technologies for Comprehensive Transport, \\ Ministry of Transport, Beijing Jiaotong University \\ 100044, Beijing, P.R. China \\ rjiang@ustc.edu.cn \\ ${ }^{3}$ Department of Civil Engineering, The University of Hong Kong \\ Pokfulam Road, Hong Kong, P.R. China \\ ${ }^{4}$ School of Mathematics and Computational Science, Anqing Normal University \\ 246133, Anqing, P.R. China
}

\begin{abstract}
The mixed flow of bicycles and pedestrians is frequently observed on bicycle-pedestrian-shared roads. Unfortunately, studies on dynamics of this kind of mixed flow are very limited. This paper reports an experimental study of this kind of mixed traffic flow with equal numbers of pedestrians and cyclists asked to walk/ride in a ring-shaped track. In the uni-/bi-directional flow scenarios, pedestrians and bicycles moved in the same/opposite direction. Under both scenarios, bicycles and pedestrians formed their own lanes. Pedestrians walked in the inner lane and cyclists rode in the outer lane. Widths of both the pedestrian lane and the bicycle lane were more uniform in bidirectional flow. The pedestrian flow rate is larger in the unidirectional flow scenario than in the bidirectional flow scenario. In contrast, at low densities, the bicycle flow rate is essentially the same under the two scenarios. When the density is large, the bicycle flow rate becomes larger in the unidirectional flow scenario. Comparing the two modes, pedestrian flow rate is smaller/larger than bicycle flow rate at small/large densities under both scenarios.
\end{abstract}

Keywords: mixed flow; pedestrian flow; bicycle flow; lane formation

\section{Introduction}

Slow traffic includes walking and cycling. Comparing to car traffic, the slow traffic has the advantage of convenience, flexibility and no pollution. The slow traffic is indispensable, and its development has effect on the whole transportation system. In recent years, the studies of pedestrian traffic flow or bicycle traffic flow have drawn wide attention in fields of physical science and engineering respectively [1 4].

In China, with the rapid development of motorization, many bicycle lanes have been converted into motor lanes. As a result, bicycles have to share the lanes with pedestrians. Chen and Xie [5] developed a traffic conflict model on urban pedestrian-bicycle road, showing that the increment of path width helps to reduce conflict intensity between cycles and pedestrians. Xie [6] analysed the traffic characteristics and conflicts on cycle-pedestrian shared path, and provided a quality description of level of service on shared-use path. Yu et al. [7] found that the service level of bicycle-pedestrian mixed traffic flow improves with the road width, and the proportion of pedestrian affects the bicycle movement significantly. However, the self-organized process and flow dynamics of the mixed flow of bicycles and pedestrians have not been investigated. In this paper, an experimental study on bicycle/pedestrian mixed traffic flow is performed. The experiment concerns both unidirectional flow and bidirectional flow scenario. The lane formation phenomenon has been observed under both scenarios. The fundamental diagrams have been investigated. 


\section{Experimental setup}

The experiments were conducted in a ring-shaped track with inner radius $8 \mathrm{~m}$ and external radius $11 \mathrm{~m} .160$ participants took part in the experiments. They were requested to walk/ride in the same direction or opposite directions. After 3-5 minutes, pedestrians were asked to turn around to walk, and cyclists went on riding anti-clockwise. Six runs of experiment were performed. Chronologically, the participant numbers were as follows: 80 pedestrians +80 bicycles (unidirectional flow first), 40 pedestrians +40 bicycles (bidirectional flow first), 70 pedestrians +70 bicycles (unidirectional flow first), 50 pedestrians +50 bicycles (bidirectional flow first), 60 pedestrians +60 bicycles (unidirectional flow first), and finally 80 pedestrians +80 cyclists (bidirectional flow first).

\section{Experimental results}

In both the unidirectional flow or bidirectional flow, pedestrians and bicycles segregate into their own lanes very quickly at any densities. Pedestrians walked in the inner lane, and cyclists rode in the outer lane (see Fig.1). In the bidirectional flow scenario, both pedestrians and cyclists avoided each other actively. Pedestrians usually walked individually, or two pedestrians walk side by side (Fig.1b). It was seldom been observed that three or more pedestrians walked side by side. Therefore, widths of both the pedestrian lane and the bicycle lane were rather uniform in the bidirectional flow. In contrast, in the unidirectional flow, pedestrians cared less about bicycles behind them. It was frequently observed that three or more pedestrians walked side by side. The red box in Fig.1a shows a typical situation where five pedestrians walked side by side. As a result, the width of pedestrian lane was not uniform. Consequently, the width of bicycle lane was not uniform, either. For example, see the situation indicated by yellow box, where bicycles occupy the whole cross section of the track.

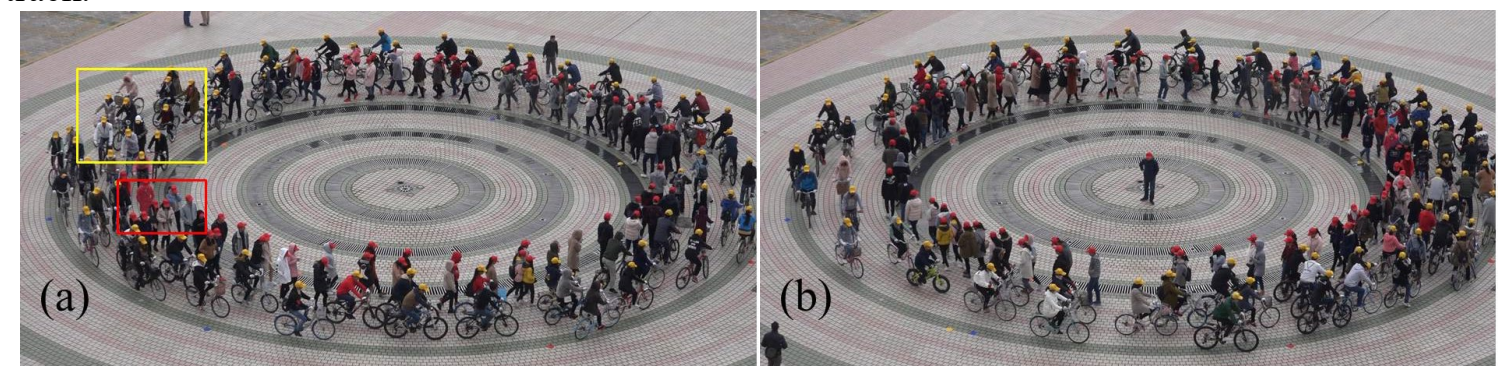

Fig. 1: Snapshots of 80 pedestrians +80 bicycles experiment. (a) unidirectional flow, (b) bidirectional flow.
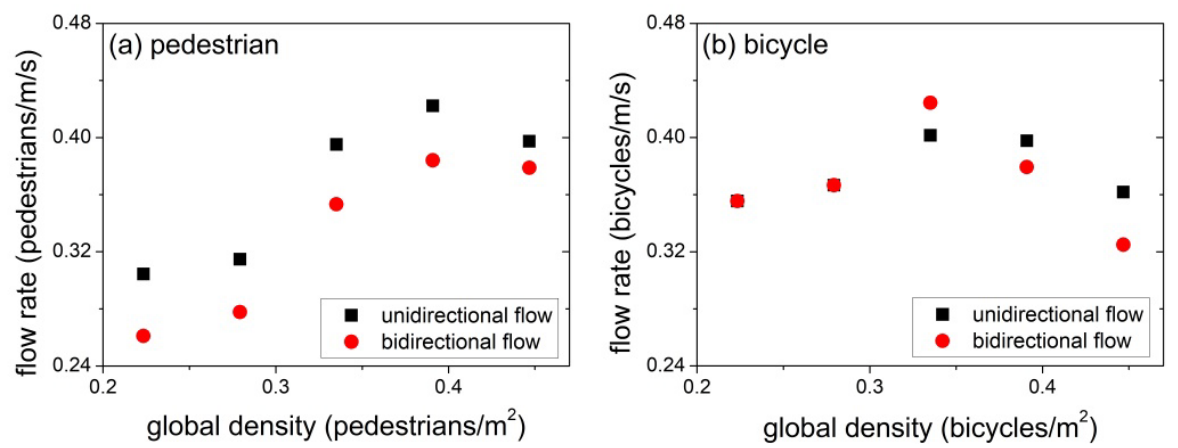

Fig. 2: Comparison unidirectional flow and bidirectional flow. (a) pedestrian flow, (b) bicycle flow in the mixed flow. Average values in 80 pedestrians +80 bicycles experiment.

Next, we study the fundamental diagram of the flow rate versus the global density. Fig.2 compares pedestrian/bicycle flow rate in the unidirectional flow with the bidirectional flow. Fig.2a shows that the maximum pedestrian flow rate was achieved at $\rho \approx 0.389$ pedestrians $/ \mathrm{m}^{2}$ under both scenarios. Moreover, the pedestrian flow rate in unidirectional flow was always larger than that in bidirectional flow. Fig. $2 b$ shows that the maximum bicycle flow rate was achieved at $\rho \approx 0.333$ 
bicycles $/ \mathrm{m}^{2}$ under both scenarios. At low densities, bicycle flow rates were essentially the same under the two scenarios. At high densities, bicycle flow rate in the unidirectional scenario became larger. However, the maximum bicycle flow in bidirectional flow was larger than that in unidirectional flow. ${ }^{1}$

Fig. 3 compares the flow rate of pedestrian and bicycle in uni- and bi-directional flow. The bicycle flow rate is larger at low densities, but smaller at high densities under both scenarios.
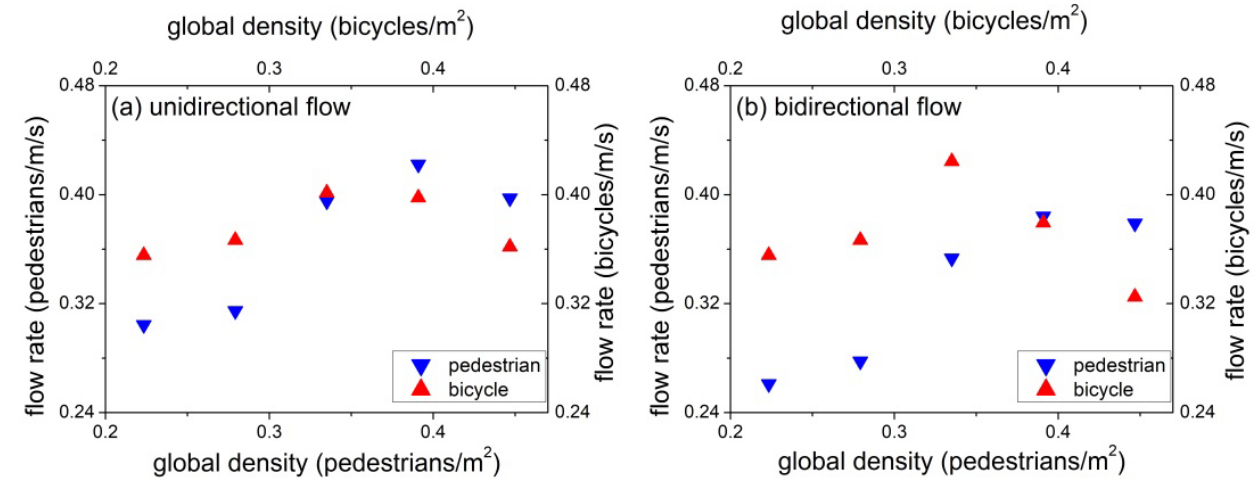

Fig. 3: Fundamental diagram of mixed flow of pedestrians and bicycles. (a) unidirectional flow, (b) bidirectional flow. Average values in 80 pedestrians +80 bicycles experiment.

\section{Conclusion}

In this paper, we have experimentally studied the mixed flow of pedestrians and bicycles. It is found that bicycles and pedestrians formed their own lanes. Widths of both pedestrian lane and bicycle lane were more uniform in bidirectional flow. The fundamental diagrams of pedestrian/bicycle flow under uni-/bi-directional scenarios have been investigated. In future work, more runs of experiments with more participants and different proportion of pedestrian/bicycle should be carried out. Moreover, it is necessary to propose models to simulate the mixed flow.

\section{Acknowledgements}

This work is supported by the National Key R\&D Program of China (No. 2017YFC0803300), the National Natural Science Foundation of China (Grants No.71621001, 71631002, 71371175), and the Beijing Natural Science Foundation (Grant No. 9172013).

\section{References}

[1] D. Helbing, I. Farkas, T. Vicsek, "Simulating dynamical features of escape panic," Nature, vol. 407, pp. 487-490, 2000.

[2] D. Helbing, L. Buzna, A. Johansson, T. Werner, "Self-organized pedestrian crowd dynamics: experiments, simulations, and design solutions," Transp.Sci., vol. 39, pp. 1-24, 2005.

[3] F.P.D. Navin, "Bicycle traffic flow characteristics: Experimental results and comparisons," ITE J., vol. 64, pp. 31-36, 1994.

[4] R. Jiang, M.B. Hu, Q.S. Wu, W.G. Song, "Traffic dynamics of bicycle flow: Experiment and modelling," Transport. Sci., vol. 51, pp. 998-1008, 2017.

[5] J. Chen, Z.Q. Xie, "Cycle traffic conflict model on urban pedestrian-bicycle paths," J. Jilin Univ., vol. 39, pp. 121-125, 2009. (in Chinese)

[6] Z.Q. Xie, "link operational characteristics and resources optimization allocation of bicycle-pedestrian shared-use paths", Nanjing: Southeast University, 2009. (Dissertation in Chinese)

[7] H. Yu, J. Chen, Z.Q. Xie, "Level of service model on urban cycle-pedestrian road," Urban Transport of China, vol. 10, pp. 75-79, 2012. (in Chinese)

\footnotetext{
1 Only one run of the experiment was performed at this density. More experiments are needed for validation.
} 